\title{
Peritoneal Reactivity Evaluation in Horses Subjected to Experimental Small Colon Enterotomy and Treated with Subcutaneous Heparin
}

\author{
Juliana de Moura Alonso, Karoline Alves Rodrigues, \\ Ana Lúcia Miluzzi Yamada, Marcos Jun Watanabe, Ana Liz Garcia Alves, \\ Celso Antonio Rodrigues, and Carlos Alberto Hussni
} School of Veterinary Medicine and Animal Science, UNESP, University Estadual Paulista, P.O. Box 560,
18618-970 Botucatu, SP, Brazil

Correspondence should be addressed to Carlos Alberto Hussni; cahussni@fmvz.unesp.br

Received 6 August 2014; Revised 17 October 2014; Accepted 17 October 2014; Published 11 November 2014

Academic Editor: Francesca Mancianti

Copyright (C) 2014 Juliana de Moura Alonso et al. This is an open access article distributed under the Creative Commons Attribution License, which permits unrestricted use, distribution, and reproduction in any medium, provided the original work is properly cited.

\begin{abstract}
Heparin is routinely administered in postoperative abdominal surgery aiming to prevent adhesions formation; however, there is no consensus indicating its effectiveness. This study evaluated the effect of heparin on peritoneal reactivity after abdominal surgery, through the association between peritoneal fluid features and ultrasonographic and laparoscopic examination. Ten adult horses were used: control group (CG) and treated group (TG). Both groups underwent laparotomy and small colon enterotomy. TG received subcutaneous heparin at $150 \mathrm{IU} / \mathrm{kg}$ every 12 hours for 5 days. The animals underwent ultrasonography and peritoneal fluid examination prior to enterotomy (M0) 12 hours (M1), 1 day (M2), 2 days (M3), 4 days (M4), 6 days (M5), 10 days (M6), and 14 days after enterotomy (M7) with laparoscopic examination being performed on the fifth postoperative day. Peritoneal inflammatory response was observed in both groups. The peritoneal fluid of TG animals showed higher echogenicity during heparin therapy. No inflammatory difference was observed between groups through peritoneal fluid features, except for the higher D-dimer concentration in CG. On laparoscopy, slightly diffuse peritoneal reactivity for both groups was observed, being higher for TG. Laparoscopy and ultrasonography association allowed detailed access to the abdominal cavity. Ultrasonography assessed the diffuse peritoneal inflammation, and laparoscopy allowed the detailed analysis of the segments. No gross beneficial reactions resulting from the use of heparin on peritoneal reactivity were observed; however, it was observed by D-dimer evaluation that the TG had less fibrin deposition, which is directly related to a lower rate of abdominal adhesions formation.
\end{abstract}

\section{Introduction}

Enterotomies are often employed for enteroliths, foreign bodies, and impacted material removal [1,2]. Surgical trauma results in peritoneal inflammation. The peritoneal response to an inflammatory stimuli of infectious nature or not is called peritonitis $[3,4]$.

After surgical trauma, peritoneal inflammation occurs concurrently with the coagulation process focusing on tissue reparative order. Thrombin is the major link between inflammation and coagulation system, since the enzyme is responsible for cleavage of circulating soluble fibrinogen to insoluble fibrin clot [5]. The coagulation process is counterbalanced by the fibrinolytic system, where the plasminogen activator (secreted by mesothelial cells, leukocytes, and tissues) acts cleaving plasminogen, the plasma protein that binds to fibrin clot to generate plasmin, a protease that acts in the lyses of fibrin [5-7].

If a persistent postsurgical inflammatory stimulus is present, an imbalance between the formation and lyses of fibrin may occur, giving rise to fibrinous adhesions which subsequently form the basis for fibrous abdominal adhesions development [8-10].

In this sense, the use of anticoagulants in order to prevent fibrin clots formation possibly inhibits adhesions formation $[10,11]$. Heparin is an acid sulfated proteoglycan 
with molecular weight variation. A small fraction of heparin is responsible for the main anticoagulant effect. This fraction binds to antithrombin III, which is a slow thrombin, plasmin, and coagulation factors inhibitor. Heparin catalyzes the antithrombin III inhibition reaction [12], by stimulating tissue plasminogen activator (tPA) and therefore enhancing plasminogen activation, which results in fibrinolysis $[12,13]$.

Due to the large size of the horse abdomen, there is no method able to evaluate fully the abdominal cavity, being necessary the combination of different methods to better evaluation. It is described that the association of ultrasonography and laparoscopy allows greater accuracy in horses digestive system diseases evaluation $[14,15]$.

Abdominal ultrasonography is a noninvasive and widely available modality that can easily integrate the examination of the abdominal cavity $[15,16]$. Laparoscopy has been expanding in abdominal disorders evaluation $[15,17,18]$.

The physical chemical and cytological evaluation of peritoneal fluid [19] is seen as test of choice for peritoneal diseases investigation and presents easy implementation, security, and wealth of information [20].

Besides this, quantification of D-dimers can also be used as a tool for peritoneal response evaluation; $\mathrm{D}$-dimer is a fragment that is released exclusively during fibrin lysis via the action of plasmin. In the serum, plasmin levels are correlated with the destruction of fibrin after hyperfibrinogenic and hypercoagulable states and serve as a marker of coagulation and fibrinolytic activity [21-23].

Few studies have been conducted using peritoneal fluid, and most of these have focused on human patients. However, measurement of the $\mathrm{D}$-dimer concentration in the peritoneal fluid of equines with severe gastrointestinal disorders demonstrated marked hyperfibrinolysis related to increased fibrin formation and degradation [22]. A D-dimer concentration $>4000 \mathrm{ng} / \mathrm{mL}$ has been established as the cut-off point for predicting poor prognosis in equines with abdominal afflictions [23].

This study aimed to evaluate heparin effect on peritoneal reactivity after laparotomy and experimental small colon enterotomy, associating peritoneal fluid features, laparoscopy, and ultrasonography examinations, since heparin is routinely used in the postoperative of abdominal surgery as a prophylactic strategy for adhesion formation inhibition, but without the existence of consistent results of its effectiveness [24].

\section{Materials and Methods}

Ten adult horses underwent a laparotomy and experimental small colon enterotomy in the standing position. Animals were divided into two groups of five animals: control group (CG) and treated group (TG). The TG immediately after surgery and every 12 hours for 5 days received heparin at a dose of $150 \mathrm{IU} / \mathrm{kg}$ subcutaneously. The CG did not receive treatment.

Prior to surgery, the right abdominal region was shaved and the horses of both groups received a combination of potassium penicillin (30.000 IU/kg intravenously), gentamicin $^{2}(6.6 \mathrm{mg} / \mathrm{kg}$ intravenously), and flunixin meglumine $(1.1 \mathrm{mg} / \mathrm{kg}$ intravenously). The procedure was performed in the standing position and under continuous infusion sedation with detomidine $(5 \mu \mathrm{g} / \mathrm{kg}$ bolus followed by continuous infusion of $20 \mu \mathrm{g} / \mathrm{kg} / \mathrm{h}$ ) associated with butorphanol $(20 \mu \mathrm{g} / \mathrm{kg}$ followed by continuous infusion of $13 \mu \mathrm{g} / \mathrm{kg} / \mathrm{h}$ ). After antisepsis, local anesthesia was performed on line incision and adjacent deep tissues with $2 \%$ lidocaine associated with vasoconstrictor.

A ten $\mathrm{cm}$ incision was performed on paralumbar fossa skin; subcutaneous tissue was divulsioned and muscle sectioned; the peritoneum was punctured and abdominal cavity accessed. Small colon identification was proceeded by direct palpation, and the further aboral segment was externalized; after delimitation and emptying of the enterotomy site segment, two coprostasis forceps were applied in order to prevent fecal flow. Subsequently two Allis clamps were applied for segment positioning and enterotomy realization in the band region; a simple separate suture pattern with 3-0 Catgut $^{7}$ was applied. After suturing, the serosal layer was subjected to abrasion with dry gauze in 50 repeated moves for each adjacent portion of the band, which results in a hemorrhagic local serosa. The segment was repositioned and abdominal wall synthesis was performed in Sultan pattern with number 2 polyglactin 910; skin suture was performed in Wolff pattern with 0 -monofilamentar nylon.

After surgery, benzathine penicillin was administered (30.000 IU/kg intramuscularly every 72 hours for 10 days), and gentamicin $(6.6 \mathrm{mg} / \mathrm{kg}$ intravenously every 24 hours for 05 days), flunixin meglumine $(1.1 \mathrm{mg} / \mathrm{kg}$ intramuscularly every 24 hours for 5 days), and antitetanus serum (10.000 UI subcutaneously in a single application) were administered for both groups.

The animals underwent ultrasonography and peritoneal fluid evaluation prior to enterotomy (M0); 12 hours (M1); 1 day (M2); 2 days (M3); 4 days (M4); 6 days (M5); 10 days (M6), and 14 days after enterotomy (M7).

The ultrasonography evaluation of small colon exam was performed with a convex transducer $(5 \mathrm{MHz}$ and $25-35 \%$ gain). To review the peritoneum and free peritoneal fluid a linear transducer (13 MHz and a gain of $80-90 \%$ ) was used.

Ultrasonography examination was performed by transabdominal via and aimed to evaluate the small colon and peritoneal fluid. The small colon was visualized in the left dorsal quadrant, using as reference the left kidney, since the small colon is usually located caudal to it and dorsal to pelvic flexure. The small colon was evaluated for its topographical location, architecture, and wall thickening.

The parietal peritoneum was evaluated in the ventral portion of the abdomen. The presence of irregularities, fibrin deposition, and peritoneal thickening from possible peritoneal inflammation were evaluated. In the same region, the quantity, echogenicity, and presence of debris on free peritoneal fluid were assessed.

For ultrasonographic findings standardization a score scheme to quantify peritoneal fluid and evaluate its echogenicity was created.

Because the dynamic feature of ultrasonography examination, it was observed that during intestinal peristalsis the 


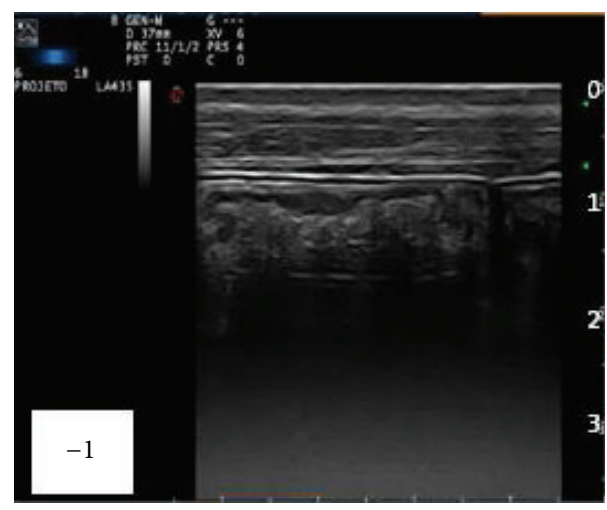

(a)

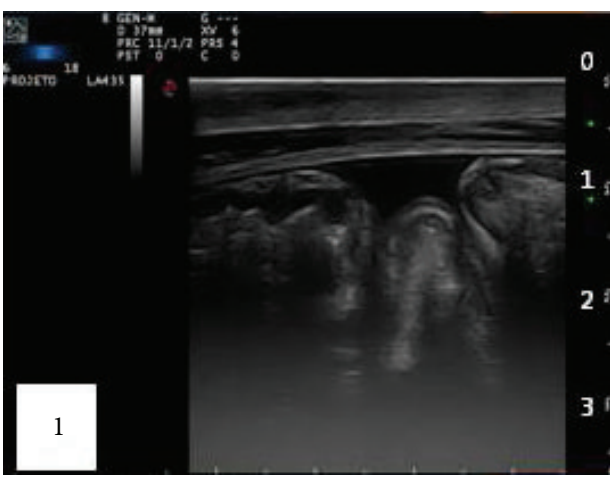

(c)

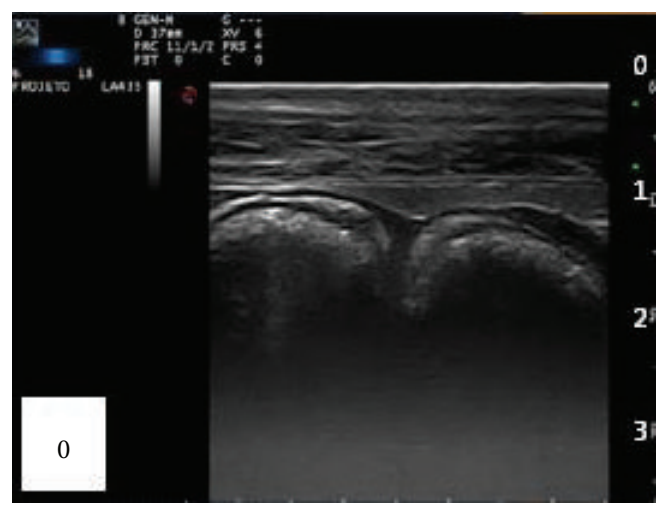

(b)

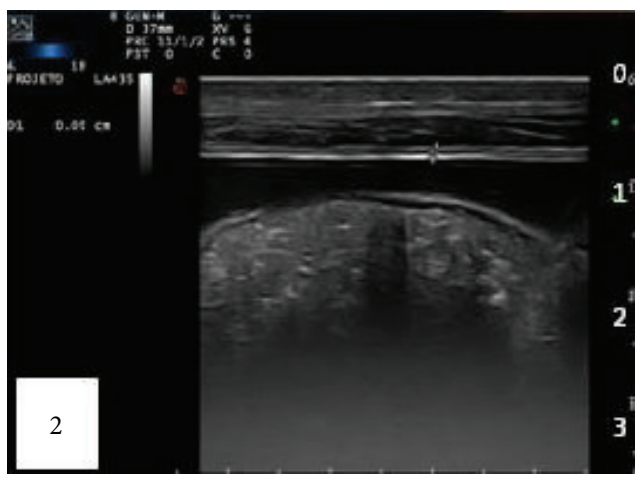

(d)

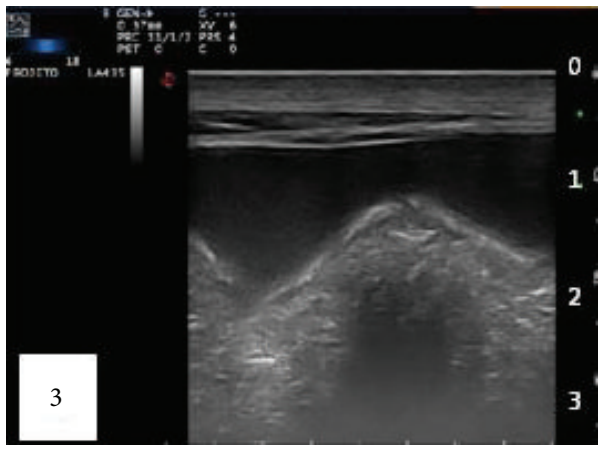

(e)

FIGURE 1: Longitudinal image of the ventral abdomen of horses from both groups, demonstrating the graduation scheme of free peritoneal fluid amount, being -1 (reduced), 0 (normal), 1 (slightly increased), 2 (moderately increased), and 3 (strongly increased).

distance between the peritoneum and the viscera adjacent to the ventral region variated, showing not to be a good evaluation tool to quantify peritoneal fluid. The association of videos findings, notes, and images obtained during ultrasonography examination was used for quantification.

The amount of free peritoneal fluid was standardized in a graduation system as follows: -1 (reduced), 0 (normal), 1 (slightly increased), 2 (moderately increased), and 3 (strongly increased), and normality was standardized as the more frequently image observed in animals prior to surgery (Figure 1).

Echogenicity was graded in a system of scores, as follows: 0 (normal), 1 (slightly increased), 2 (moderately increased), and 3 (moderately increased, associated with the presence of cellular debris), and normality was established as the appearance of liquid prior to surgical procedure (Figure 2).
The peritoneal fluid samples were collected at the most ventral region of the abdomen using a $40 \times 12 \mathrm{~mm}$ hypodermic needle and tubes containing $3.8 \%$ sodium citrate at a 9:1 (liquid: anticoagulant) ratio $(\mathrm{v} / \mathrm{v})$ for $\mathrm{D}$-dimer evaluation, EDTA tubes for cytology count, and tubes with no anticoagulant for physicochemical tests. The samples for the D-dimer were centrifuged for 15 minutes immediately after collection, using a $1000 \mathrm{G}$ rotation. D-dimer analysis was performed using the latex agglutination test, according to the manufacturer's instructions.

Fifteen days after the initial surgery, a new surgical approach was performed. The animals received $700 \mathrm{~g}$ of magnesium suphate 36 hours before the procedure and were subjected to 24 hours fasting, in order to small colon emtying and easier handling of the segments. Prior to surgery, the 


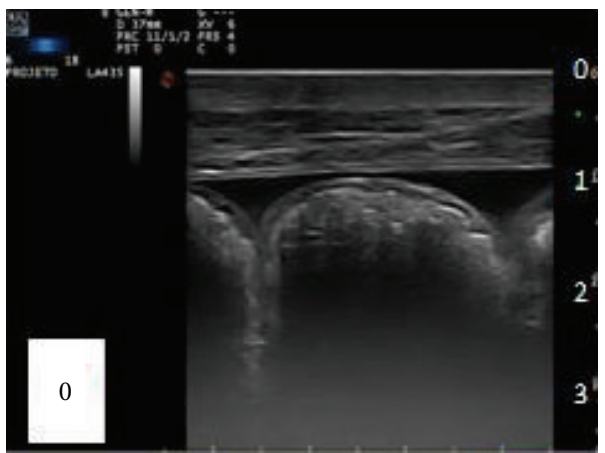

(a)

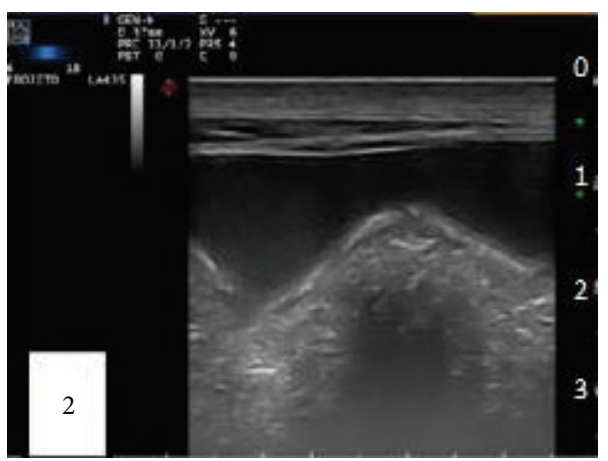

(c)

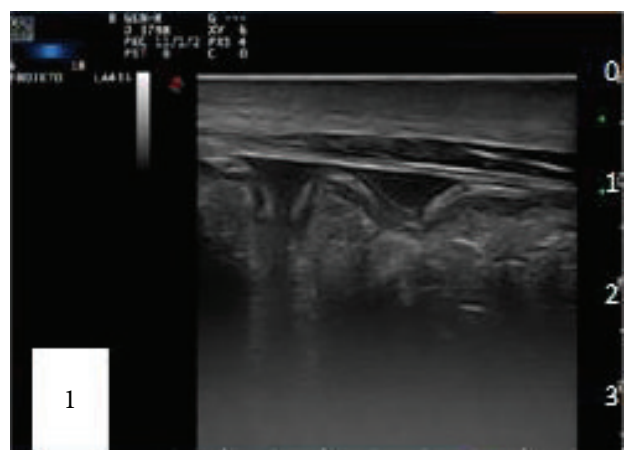

(b)

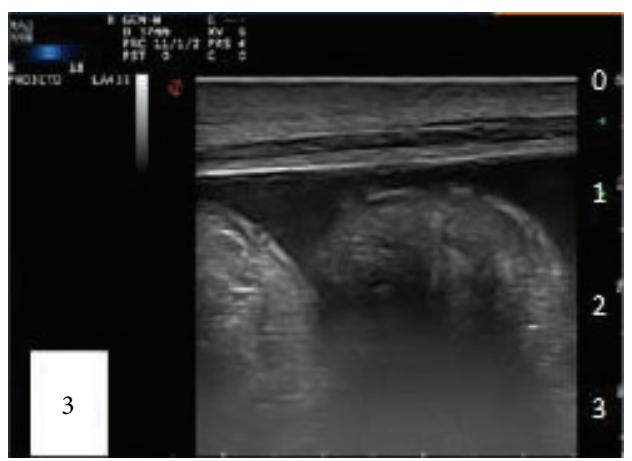

(d)

Figure 2: Longitudinal image of horses ventral abdomen from both groups. Demonstrative Scheme graduation of peritoneal fluid echogenicity, being 0 (normal), 1 (slight increase), 2 (moderate increase), and 3 (moderate increase associated with the presence of cellular debris).

left abdomen was shaved. Rectal palpation was proceeded in order to assess the anatomic topography at the site of cannulae insertion, avoiding iatrogenic punctures. The procedure was performed in standing position with identical sedation protocol to that used in laparotomy.

Laparoscopic equipment was composed of electronic insufflator $\mathrm{CO}_{2}$, Light Source Xenon $180 \mathrm{~W}$, illumination cable, microcamera with processor, rigid endoscope $(10 \mathrm{~mm}$ diameter, $57 \mathrm{~cm}$ length of 0 and 30 degrees) cannula EndoTIP TM (10 mm), laparoscopic and conventional surgical instruments, TV monitor, image recording system comprising a notebook, and a capture plate.

The surgical access area underwent local anesthesia with $2 \%$ lidocaine associated with vasoconstrictor. Antisepsis was performed; the skin incision was approximately $2 \mathrm{~cm}$; the access was created through the introduction of videoassisted cannula (EndoTIP TM) with rigid endoscope inside. The pneumoperitoneum was induced. The abdominal pressure was maintained during the procedure between 12 and $15 \mathrm{mmHg}$, varying according to each animal supported volume. A systematic evaluation of abdominal cavity was established to perform a complete scan of structures amenable to evaluation through the left observation.

After abdominal cavity evaluation, two new anesthetic blocks points of approximately $1.5 \mathrm{~cm}$ where performed for cannulas insertion $(11 \mathrm{~mm})$; atraumatic intestinal graspers (Babcock) were introduced. A detailed evaluation of the small colon and enterotomy region was performed with Babcock forceps, supporting mesocolon dorsally; the optical was advanced to the right side of the abdomen in order to observe the laparotomy wound region. The images were stored for later evaluation.

The graspers were removed and the safety valves of the cannulas were opened allowing the release of the $\mathrm{CO}_{2}$; the cannulas were removed and finally withdrew the EndoTIP by performing rotational movements that promoted muscle layers approximation; the skin synthesis was performed in simple separated standard with nylon 0 .

In order to facilitate the images evaluation scores of diffuse peritoneal reactivity were created, being 0 (no diffuse reactivity), 1 (mild diffuse reactivity), and 2 (moderately diffuse reactivity) (Figure 3).

The Wilcoxon test [25] was used to compare the median of each variable between the study groups. Later, comparisons between groups were performed at all times. Statistical analysis was performed with the PROC Nparlway [26] and statistical significance was defined as $P<0.05$.

\section{Results}

The applied ultrasonographic methodology allowed the evaluation of small colon segments in approximately $85 \%$ of patients in different times, and possibly among these, only in a small proportion, the segment subjected to enterotomy was 


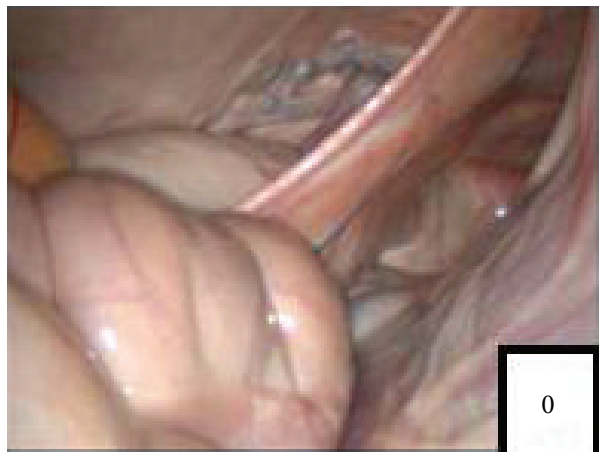

(a)

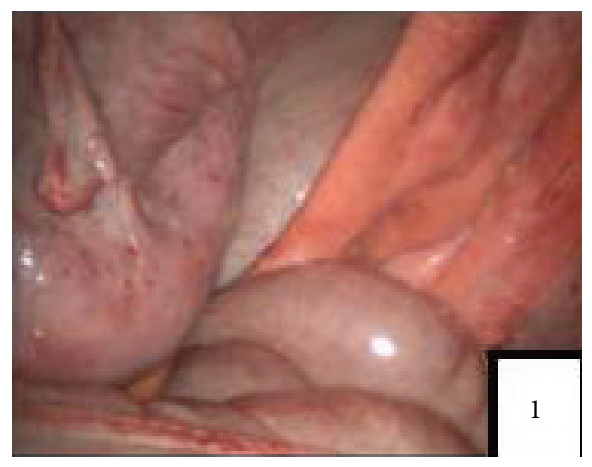

(b)

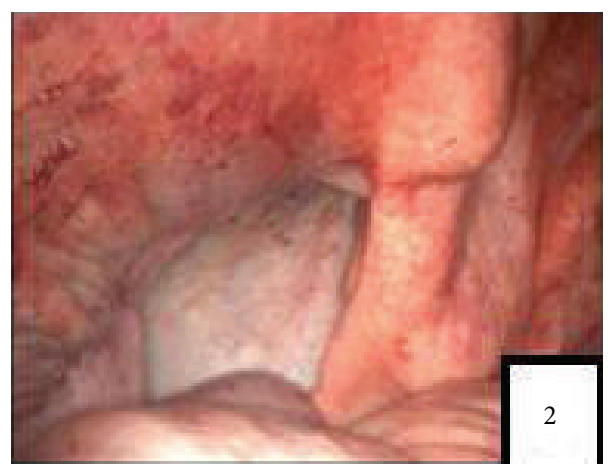

(c)

Figure 3: Score system of diffuse peritoneal reactivity, being 0 (no diffuse reactivity), 1 (mild diffuse reactivity), and 2 (moderate diffuse reactivity).

found, being rarely observed segments with wall thickening and architecture regularity loss.

Ventral abdomen ultrasonograph examination allowed the assessment of peritoneal fluid quantity, echogenicity, homogeneity, flocculation, and fibrin presence.

No difference between groups was observed for the amount of free peritoneal fluid; however an individual variation among animals was observed. Based on employed graduation scale it was found that animals maintained their individual volume regardless of the surgical procedure. After the 10th day after surgery there was a slight increase in the amount of free fluid in both groups, which is represented by the increase of one score in scale.

Relying on the proposed graduation scale, peritoneal echogenicity increased after 12 hours of surgery for both groups. Increased echogenicity persisted until the 14th day, however, with progressive reduction after the 6 th day postoperatively. Although there was no significant difference between groups, the TG showed persistence of increased echogenicity in relation to the CG (Table 1).

Fibrin clots and irregularities in the parietal peritoneum were present between the 4 th and 6 th day in both groups.

After 12 hours of surgery, all peritoneal fluid samples presented red color; for TG the red color was maintained throughout the treatment period. Significant difference was obtained between groups $(P<0.0470)$, with $48 \%$ of red samples in CG and $72 \%$ for TG. In subsequent analysis, peritoneal fluid acquired gradually decreasing turbidity and coloration tends to be normal.
TABLE 1: Peritoneal fluid echogenicity evolution on ultrasonograph using the employed score system (animals percentage).

\begin{tabular}{lccccc}
\hline Moment & Group & Score 0 & Score 1 & Score 2 & Score 3 \\
\hline \multirow{2}{*}{ M0 } & CG & $100 \%$ & & & \\
& TG & $100 \%$ & & & \\
\hline \multirow{2}{*}{ M1 } & CG & & & $80 \%$ & $20 \%$ \\
& TG & & & $80 \%$ & $20 \%$ \\
\hline \multirow{2}{*}{ M2 } & CG & & & $100 \%$ & \\
& TG & & & $80 \%$ & $20 \%$ \\
\hline \multirow{2}{*}{ M3 } & CG & & & $100 \%$ & \\
& TG & & & $100 \%$ & \\
\multirow{2}{*}{ M4 } & CG & & $60 \%$ & $40 \%$ & \\
& TG & & & $100 \%$ & \\
\multirow{2}{*}{ M5 } & CG & $20 \%$ & $60 \%$ & $20 \%$ & \\
& TG & & $40 \%$ & $60 \%$ & \\
\multirow{2}{*}{ M6 } & CG & $60 \%$ & $20 \%$ & $20 \%$ & \\
& TG & & $100 \%$ & & \\
\multirow{2}{*}{ M7 } & CG & $80 \%$ & $20 \%$ & & \\
& TG & & $100 \%$ & & \\
\hline
\end{tabular}

No significant difference between groups. 0: normal, 1: mild increase in echogenicity, 2: moderate increase in echogenicity, and 3: moderate increase in echogenicity accompanied by the presence of fibrin and cellular debris.

After laparotomy, there were an increased number of neutrophils, mononuclear cells, lymphocytes, and macrophages in both groups peritoneal fluid; all parameters increased in 
TABLE 2: Comparison of the total peritoneal RBC count between the control (GC) group and the treated group (TG) at different times (RBCs $/ \mu \mathrm{L})$.

\begin{tabular}{lcccccc}
\hline \multirow{2}{*}{ Moment } & \multicolumn{3}{c}{ CG } & & & TG \\
& Median & Q1 & Q3 & Median & Q1 & Q3 \\
\hline M0 & 6350 & 5550 & 9300 & 1650 & 340 & 4750 \\
M1 & 390000 & 218400 & 475000 & 1306500 & 301150 & 2532600 \\
M2 & 681050 & 226125 & 1315550 & 2632600 & 1240675 & 3085350 \\
M3 & $\mathbf{8 8 0 0 0}^{*}$ & 63800 & 160800 & $\mathbf{5 7 2 8 5 0}^{*}$ & 462300 & 1708500 \\
M4 & $\mathbf{3 0 1 5 0 *}^{*}$ & 8800 & 39600 & $\mathbf{3 2 1 6 0 0 *}^{*}$ & 261300 & 522600 \\
M5 & 8800 & 5500 & 12600 & 83125 & 25550 & 140700 \\
M6 & 7850 & 6050 & 10950 & 48825 & 15800 & 60300 \\
M7 & 33900 & 12700 & 70350 & 9550 & 4200 & 44500 \\
\hline
\end{tabular}

${ }^{*}$ Significant difference between groups $(P<0.05)$. Q1: first quartile; Q3: third quartile.

TABLE 3: Comparison of the peritoneal D-dimer concentrations between the CG and TG at different time-points.

\begin{tabular}{lcccccc}
\hline \multirow{2}{*}{ Time-point } & \multicolumn{5}{c}{ Control group } & \multicolumn{3}{c}{ Treated group } \\
& Median & Q1 & Q3 & Median & Q1 & Q3 \\
\hline M0 & 0 & 0 & 0 & 0 & 0 & 0 \\
M1 & 32000 & 1000 & 32000 & 8000 & 8000 & 16000 \\
M2 & 8000 & 8000 & 8000 & 4000 & 2000 & 32000 \\
M3 & 8000 & 4000 & 16000 & 2000 & 1000 & 8000 \\
M4 & 4000 & 4000 & 8000 & 2000 & 2000 & 8000 \\
M5 & 2000 & 1000 & 4000 & 500 & 0 & 1000 \\
M6 & 4000 & 500 & 4000 & 500 & 500 & 1000 \\
M7 & 500 & 500 & 2000 & 0 & 0 & 2000 \\
\hline
\end{tabular}

There was no significant difference between groups. Q1: first quartile; Q3: third quartile.

relation to the normal range [27]; however, no significant difference was observed between the groups.

For peritoneal RBC a significant difference between the groups was observed $(P<0.0365)$ when all times were combined (median value: CG-33900 and TG-140700 $\mathrm{RBCs} / \mu \mathrm{L})$ and at M3 $(P<0.0358)$ and M4 $(P<0.0122)$ (Table 2).

The baseline values of $\mathrm{D}$-dimer were below the reference values ( 4.0 to $88.0 \mathrm{ng} / \mathrm{mL}$ ) [22] with detected concentrations $<0.5 \mathrm{ng} / \mathrm{mL}$. After surgery, considerably higher values were obtained for the CG, with median values of $4000 \mathrm{ng} / \mathrm{mL}$ (CG) and $1500 \mathrm{ng} / \mathrm{mL}$ (TG). However, there were no significant differences $(P=0.0745)$ between groups when all timepoints were combined or not (Table 3).

No changes in visceral topography were observed on laparoscopic exam. The quantity and quality of peritoneal fluid evaluated macroscopically were compatible with ultrasonographic findings and did not differ between groups.

During small colon manipulation with atraumatic forceps, it was observed that adjacent regions to enterotomy had become covered with a thin layer of fibrinous exudate and presented reactive and friable to manipulation, rapidly becoming bleeding in both groups, but with greater intensity in TG.
Although there was no significant difference in diffuse peritoneal reactivity between groups, there was evidently greater reactivity in TG. CG showed $60 \%$ of score $0,20 \%$ of score 1 , and $20 \%$ of score 2 and TG showed $60 \%$ of score 1 and $40 \%$ of score 2 .

Invariably, the region of the enterotomy to both groups was covered by fibrin. On the 15th day after enterotomy the suture performed with catgut was preserved. No difference between groups was observed for fibrin deposition over enterotomy region (Figure 4).

\section{Discussion}

Moore and Hinchcliff [12] described that an effective heparin dosage will be around $150 \mathrm{UI} / \mathrm{Kg}$ every 12 hours and suggest that a decreasing regimen should be used to avoid side effects, as anemia, hemorrhage, thrombocytopenia, and painful swelling at injection sites. In spite of no reduction on dosage during our treatment we observed no hemorrhage and a transient anemia and thrombocytopenia, which were rapidly reversible after discontinuation of treatment. Heparin probably results in microcirculation accumulation of $\mathrm{RBC}$ and platelets, which explains the rapid recovery of such amounts [12, 28-30].

Although transrectal ultrasonography is the most appropriate method for evaluating small colon [16], transabdominal route was chosen to avoid interference on enterotomy healing. The applied methodology did not allow the access to the small colon in all the exams. Small colon has been accessed in approximately $85 \%$ of patients in different times. Furthermore, among these $85 \%$, only in a small proportion changes in the evaluated segments were observed; probably the applied methodology has not been adequate to evaluate the specific segment subjected to enterotomy.

Increased echogenicity observed 12 hours after surgery in both groups was assigned to cellular migration in response to abdominal inflammatory process and intracavitary surgical bleeding. The difference observed between groups for echogenicity possibly occurred due to heparin treatment, which resulted in maintenance of the displacement of RBCs for peritoneal fluid, since the high erythrocyte count, macroscopic aspect, and a gradual decreased in echogenicity for TG after the suspension of treatment.

Despite the fact that the difference in echogenicity did not show statistical significance between groups, it was observed that the TG showed higher echogenicity. Probably a study with a large number of animals should demonstrate statistical difference.

Postoperative peritonitis, fibrin presence, and peritoneal irregularities observed during this study corroborate with literature information that reports increased echogenicity due to high cellularity, loss of homogeneity, fibrin deposition, and presence of cellular debris [16, 31].

Although TG had presented higher peritoneal fluid echogenicity, it cannot be inferred by ultrasonographic examination that these animals had higher peritoneal reactivity, since the high liquid cellularity corresponded mainly to erythrocytes, with no difference between groups for 

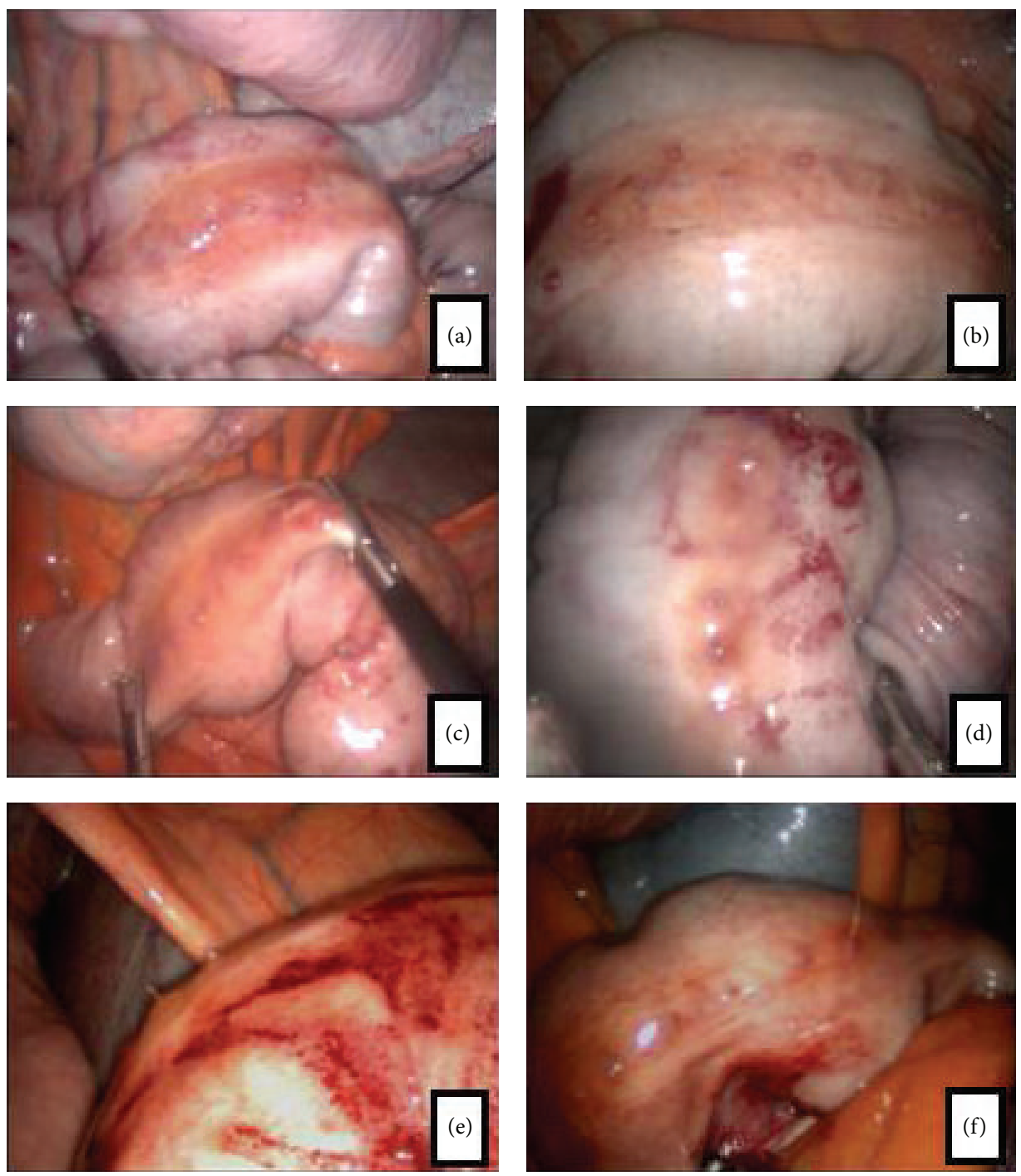

FIGURE 4: Laparoscopic image of small colon region subjected to enterotomy. (a), (b), and (c) control group (small colon enterotomy region covered by fibrin); (d), (e), and (f) treated group (small colon enterotomy region covered with fibrin; segments presenting hemorrhagic soon after manipulation).

the presence of fibrin, cellular debris, and thickening of the peritoneum and intestinal walls.

Ultrasonography allowed peritoneal cavity inflammation and bleeding due to heparin treatment identification but did not allow detailed tracing of small colon. Transabdominal ultrasound use may have influenced the sensitivity to detect small colon changes.

Although there was no significant difference in the Ddimer concentration between groups, higher values tended to be observed for animals in the CG, which is consistent with the notion that abdominal surgery results in a state of hyperfibrogenesis and hyperfibrinolysis [22]. Because the $\mathrm{D}$-dimer fragment is released exclusively during fibrin lysis by plasmin, it was inferred that the animals in the heparintreated group, despite undergoing the same surgical procedure, had lower D-dimer concentrations due to reduced formation and deposition of fibrin and consequently lower cleavage rates of fibrin.
D-dimer values are directly related to poor prognosis and high mortality; the indication for the use of heparin in hypercoagulable states and after abdominal surgery in equines was strengthened by the results of our study.

Although the animals in the present study did not show abdominal adhesions, our results highlight the potential use of heparin for prophylaxis in cases of abdominal adhesions. However, further clinical studies should evaluate the use of heparin in equines suffering from colic syndrome and those subjected to laparotomy.

Laparoscopy was performed through the left abdominal approach, a procedure which according to Galuppo et al. [17] allows small colon visualization. No visceral topography changes were observed in evaluated animals, being the structures localized as described by Galuppo et al. [17] and Silva et al. [32]. It was observed that both groups had mild diffuse peritoneal reactivity; however TG showed higher reactivity. Moreover, these animals responded to visceral manipulation 
with more frequent formation of hemorrhagic areas at the contact points with the graspers. It was expected that the TG presented less fibrin deposition over enterotomy region; however no gross difference between groups was observed.

Laparoscopic examination allowed a detailed abdominal cavity and enterotomy area analysis, demonstrating no significant difference on fibrin deposition over enterotomy region; however without statistical significance, more diffuse peritoneal reactivity to visceral manipulation on TG was observed.

The association of peritoneal fluid evaluation, laparoscopy, and ultrasonography allowed detailed access to the abdominal cavity. Ultrasonography and peritoneal fluid evaluation allowed the assessment of diffuse peritoneal inflammation, while laparoscopy allowed the evaluation of peritoneal inflammation and a detailed analysis of the segment subjected to enterotomy.

No gross beneficial reactions resulting from the use of heparin on peritoneal reactivity were observed; however, it was observed by D-dimer evaluation that the TG had less fibrin deposition, which is directly related to a lower rate of abdominal adhesions formation.

\section{Conflict of Interests}

The authors declare that there is no conflict of interests regarding the publication of this paper.

\section{Acknowledgments}

The authors would like to acknowledge São Paulo Research Foundation for financial support (2011/23359-9) and CNPq.

\section{References}

[1] C. A. Duarte, J. W. Cattelan, A. C. Alessi, P. P. Valente, A. C. Aita, and L. Rasera, "Enterorrafias em plano aposicional convencionale com adesivo a base de cianoacrilato no colón descendente de equinos," Ciência Rural, vol. 32, no. 4, pp. 595601, 2002.

[2] T. G. Montello, J. F. C. Castro Junior, V. P. Santos, E. C. S. Christo, and A. P. F. Silva Filho, "Alterações hematológicas observadas em equinos submetidos a laparotomia em estação e enterotomia do colon menor," Acta Scientiae Veterinariae, vol. 32, no. 3, pp. 201-205, 2004.

[3] A. M. Trent, "The peritoneum and peritoneal cavity," in The Horse Diseases E Clinical Management, C. N. Kobluk, Ed., pp. 373-404, WB Saunders, Philadelphia, Pa, USA, 1995.

[4] M. H. Hillyer and C. J. Wright, "Peritonitis in the horse," Equine Veterinary Education, vol. 9, no. 3, pp. 136-142, 1997.

[5] R. Mitchell, V. Kumar, N. Fausto, A. K. Abbas, and J. Aster, "Inflamação aguda e crônica," in Robbins \& Cotran. Fundamentos de Patologia. Bases Patológicas das doenças, pp. 29-54, 7th edition, 2005.

[6] L. Holmdahl, B. Risberg, D. E. Beck et al., "Adhesions: pathogenesis and prevention-panel discussion and summary," The European Journal of Surgery. Supplement, vol. 577, pp. 56-62, 1997.
[7] M.-L. Ivarsson, P. Falk, and L. Holmdahl, "Response of visceral peritoneum to abdominal surgery," British Journal of Surgery, vol. 88, no. 1, pp. 148-151, 2001.

[8] Y. C. Cheong, S. M. Laird, T. C. Li, J. B. Shelton, W. L. Ledger, and I. D. Cooke, "Peritoneal healing and adhesion formation/reformation," Human Reproduction Update, vol. 7, no. 6, pp. 556-566, 2001.

[9] A. N. Imudia, S. Kumar, G. M. Saed, and M. P. Diamond, "Pathogenesis of intra-abdominal and pelvic adhesion development," Seminars in Reproductive Medicine, vol. 26, no. 4, pp. 289-297, 2008.

[10] B. C. Ward and A. Panitch, "Abdominal adhesions: current and novel therapies," Journal of Surgical Research, vol. 165, no. 1, pp. 91-111, 2011.

[11] R. B. Eggleston and P. O. E. Mueller, "Prevention and treatment of gastrointestinal adhesions," Veterinary Clinics of North America - Equine Practice, vol. 19, no. 3, pp. 741-763, 2003.

[12] B. R. Moore and K. W. Hinchcliff, "Heparin: a review of its pharmacology and therapeutic use in horses," Journal of Veterinary Internal Medicine, vol. 8, no. 1, pp. 26-35, 1994.

[13] P. Andrade-Gordon and S. Strickland, "Interaction of heparin with plasminogen activators and plasminogen: effects on the activation of plasminogen," Biochemistry, vol. 25, no. 14, pp. 4033-4040, 1986.

[14] C. Ragle, "Roundtable discussion: laparoscopy in Equine Practice: part 1," Equine Practice, vol. 19, no. 9, pp. 7-15, 1997.

[15] E. Mueller and K. Epstein, "A view into the big "black box": peering through the window with ultrasound and laparoscopy," Equine Veterinary Education, vol. 21, no. 1, pp. 31-35, 2009.

[16] V. B. Reef, M. Whittier, and L. G. Allam, "Sonographic evaluation of the adult abdomen," Clinical Techniques in Equine Practice, vol. 3, no. 3, pp. 294-307, 2005.

[17] L. D. Galuppo, J. R. Snyder, and J. R. Pascoe, "Laparoscopic anatomy of the equine abdomen," The American Journal of Veterinary Research, vol. 56, no. 4, pp. 518-531, 1995.

[18] C. L. Smith, B. A. Dowling, and A. J. Dart, "Recent advances in equine abdominal surgery," Veterinary Journal, vol. 170, no. 1, pp. 41-51, 2005.

[19] D. R. Hodgson and R. J. Rose, Manual of Equine Practice, W.B. Saunders, Philadelphia, Pa, USA, 1993.

[20] E. P. Tulleners, "Complications of abdominocentesis in the horse," Journal of the American Veterinary Medical Association, vol. 182, no. 3, pp. 232-234, 1983.

[21] T. Stokol, H. N. Erb, L. de Wilde, S. J. Tornquist, and M. Brooks, "Evaluation of latex agglutination kits for detection of fibrin(ogen) degradation products and D-dimer in healthy horses and horses with severe colic," Veterinary Clinical Pathology, vol. 34, no. 4, pp. 375-382, 2005.

[22] M. A. Delgado, L. Monreal, L. Armengou, J. Ríos, and D. Segura, "Peritoneal D-dimer concentration for assessing peritoneal fibrinolytic activity in horses with colic," Journal of Veterinary Internal Medicine, vol. 23, no. 4, pp. 882-889, 2009.

[23] C. Cesarini, L. Monreal, L. Armengou, M. Á. Delgado, J. Ríos, and E. Jose-Cunilleras, "Association of admission plasma Ddimer concentration with diagnosis and outcome in horses with colic," Journal of Veterinary Internal Medicine, vol. 24, no. 6, pp. 1490-1497, 2010.

[24] K. M. Claunch and P. O. E. Mueller, "Treating intra-abdominal adhesions: the surgeon's dilemma," Equine Veterinary Education, vol. 24, no. 11, pp. 552-555, 2012. 
[25] M. Pagano and K. Gauvreau, Principles of Biostatistics, Duxbury/Thomson Learning, Pacific Grove, Calif, USA, 2000.

[26] SAS Institute, SAS/STAT 9.2 User's Guide, SAS Institute, Cary, NC, USA, 2nd edition, 2009.

[27] M. M. Neves, J. R. A. P. Marques, G. E. S. Alves, and E. P. Faria, "Valores referenciais da análise do líquido peritoneal de equinos sadios," Ciência Rural, vol. 30, no. 5, pp. 809-811, 2000.

[28] E. A. Mahaffey and J. N. Moore, "Erythrocyte agglutination associated with heparin treatment in three horses," Journal of the American Veterinary Medical Association, vol. 189, no. 11, pp. 1478-1480, 1986.

[29] J. N. Moore, E. A. Mahaffey, and M. Zboran, "Heparin-induced agglutination of erythrocytes in horses," American Journal of Veterinary Research, vol. 48, no. 1, pp. 68-71, 1987.

[30] H. Gerhards, "Low dose calcium heparin in horses: plasma heparin concentrations, effects on red blood cell mass and on coagulation variables," Equine Veterinary Journal, vol. 23, no. 1, pp. 37-43, 1991.

[31] A. Desrochers, Abdominal Ultraultrasonography of Normal and Colicky Adult Horses, American Association of Equine Practitioners, Québec, Canada, 2005.

[32] L. C. L. C. Silva, A. L. V. Zoppa, and D. A. Hendrickson, "Equine diagnostic laparoscopy," Journal of Equine Veterinary Science, vol. 28 , no. 4 , pp. $247-254,2008$. 

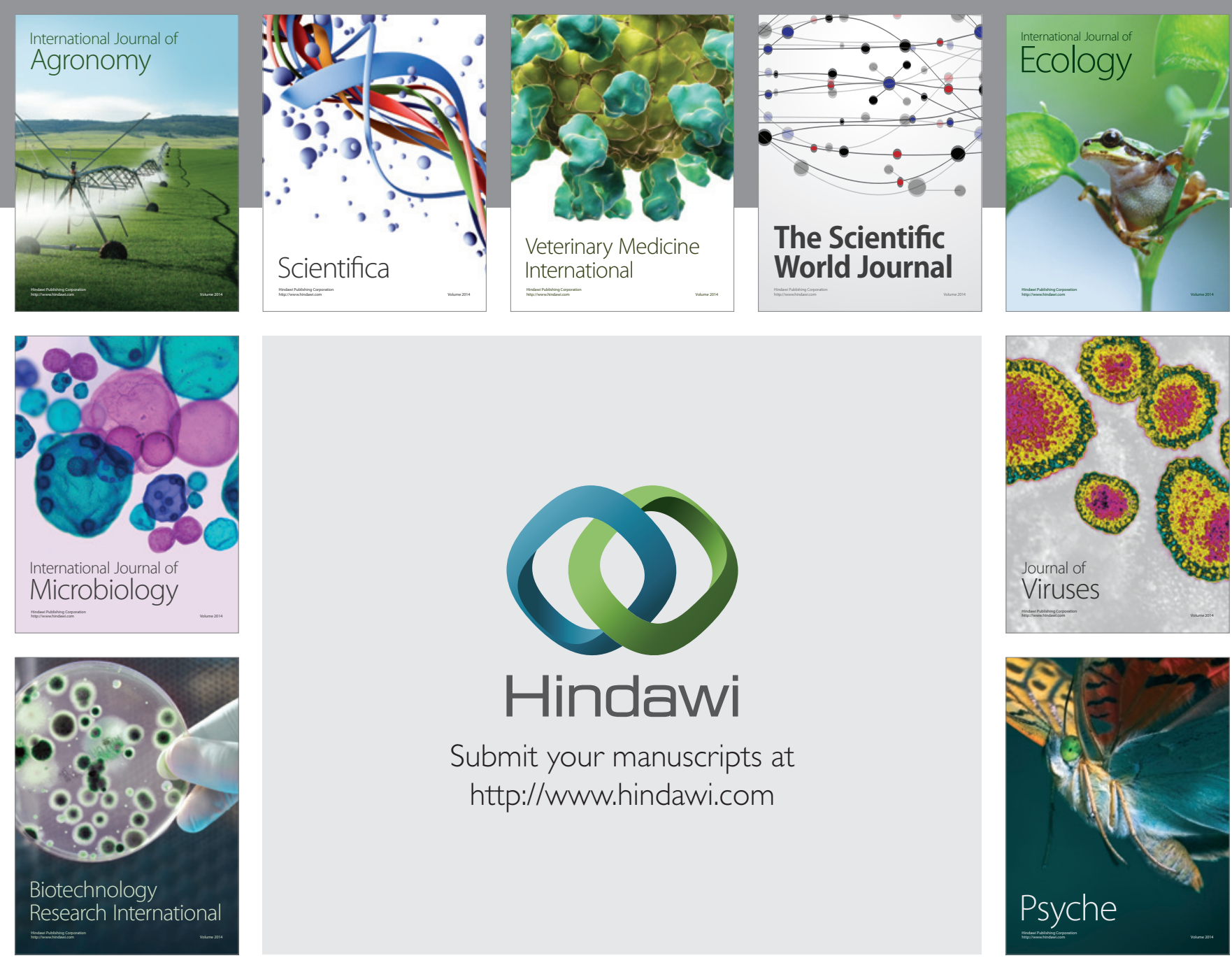

Submit your manuscripts at http://www.hindawi.com
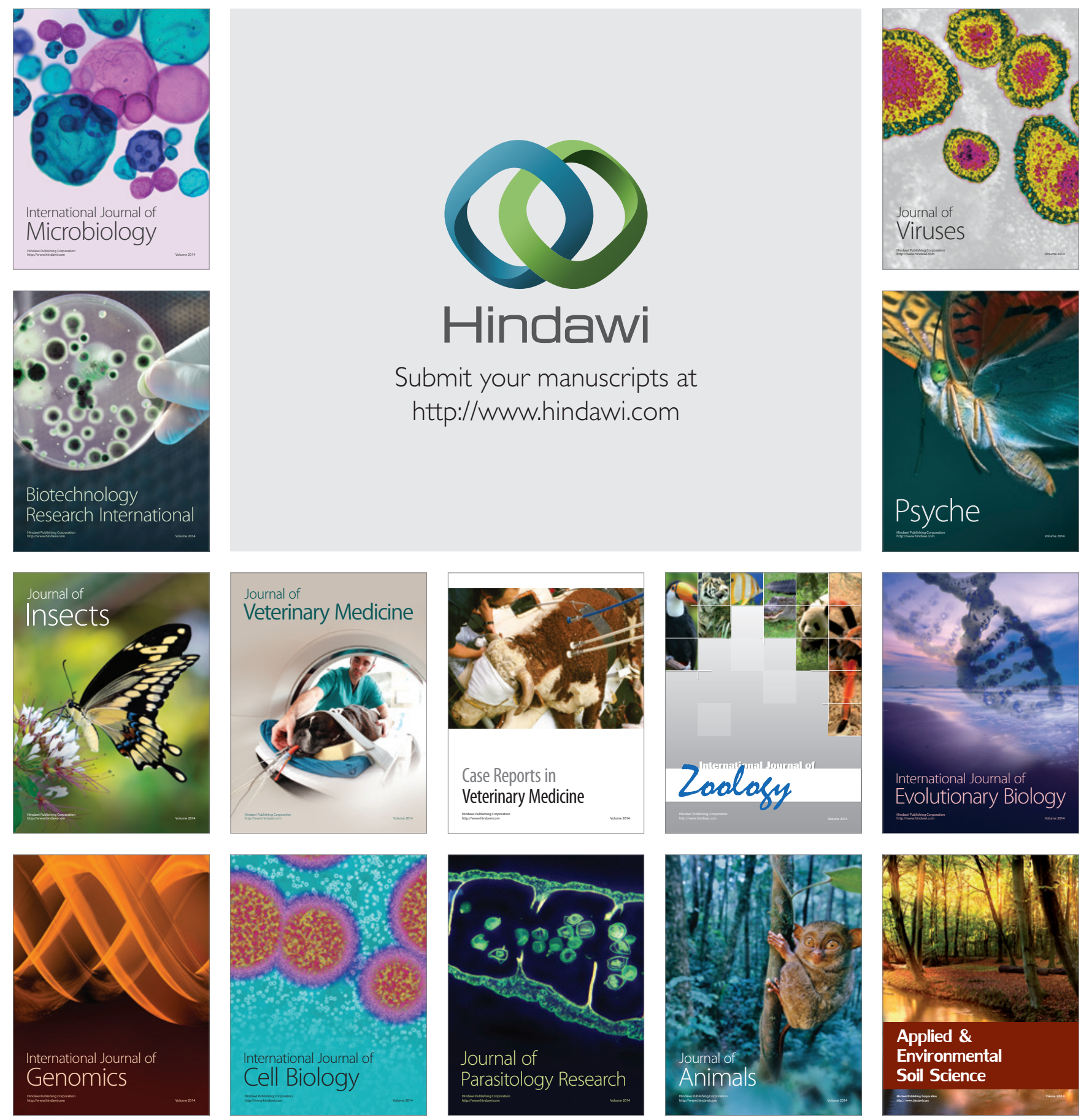